\title{
Clusters of male and female Alzheimer's disease patients in the Alzheimer's Disease Neuroimaging Initiative (ADNI) database
}

\author{
Dragan Gamberger • Bernard Ženko • Alexis Mitelpunkt • \\ Netta Shachar • Nada Lavrač
}

Received: 13 November 2015/Accepted: 21 January 2016/Published online: 30 March 2016

(C) The Author(s) 2016. This article is published with open access at Springerlink.com

\begin{abstract}
This paper presents homogeneous clusters of patients, identified in the Alzheimer's Disease Neuroimaging Initiative (ADNI) data population of 317 females and 342 males, described by a total of 243 biological and clinical descriptors. Clustering was performed with a novel methodology, which supports identification of patient subpopulations that are homogeneous regarding both clinical and biological descriptors. Properties of the constructed clusters clearly demonstrate the differences between female and male Alzheimer's disease patient groups. The major difference is the existence of two male subpopulations with unexpected values of intracerebral and whole brain volumes.
\end{abstract}

For the Alzheimer's Disease NeuroimagingInitiative.

Data used in this work were obtained from the Alzheimer's Disease Neuroimaging Initiative (ADNI) database (adni.loni.usc.edu). As such, the investigators within the ADNI contributed to the design and implementation of ADNI and/or provided data but did not participate in analysis or writing of this report. A complete listing of ADNI investigators can be found at http://adni.loni.usc.edu/wp-content/ uploads/how_to_apply/ADNI_Acknowledgement_List.pdf.

D. Gamberger $(\bowtie)$

Ruđer Bošković Institute, Zagreb, Croatia

e-mail: dragan.gamberger@irb.hr

B. Ženko

Jožef Stefan Institute, Ljubljana, Slovenia

A. Mitelpunkt · N. Shachar

Tel Aviv University, Tel Aviv, Israel

N. Lavrač

Jožef Stefan Institute, Ljubljana, Slovenia

N. Lavrač

University of Nova Gorica, Nova Gorica, Slovenia
Keywords Alzheimer's disease - Clustering · Female and male subpopulations

\section{Introduction}

A key issue in understanding of the Alzheimer's disease (AD) is the recognition of relations between clinical characteristics of patients and their biological properties that can be objectively measured. Some recent studies [1] suggest the existence of different AD subtypes, and it may be expected that the identification of relevant relations is potentially easier for $\mathrm{AD}$ subtypes than for the complete $\mathrm{AD}$ population. Additionally, segmentation of the $\mathrm{AD}$ population may enable comparative evaluation of subpopulations of $\mathrm{AD}$ patients, potentially leading to a better understanding of their distinguishing properties.

An important characteristic of the proposed approach applied in this work is that clustering is performed separately for male and female populations, and that the generated patient clusters are homogeneous both in terms of clinical and biological properties. The results are relevant for identification of gender-specific properties of patients that have problems with dementia. Also, the results clearly support the conclusion that there are significant genderrelated differences among $\mathrm{AD}$ patients [2].

Development of our clustering methodology has been motivated by the recently introduced approaches of redescription mining [3] and multi-view learning [4]. We give a detailed presentation of the proposed multi-layer clustering algorithm (MLC), since we believe that this methodology can be useful also in other medical applications. The algorithm is implemented as a web application and is easy to use. It is publicly available at http://rr.irb.hr/MLC/. 
Applications of data clustering are very good examples of interactive data mining approaches [5] because evaluation of the quality of obtained clusters is only possible in the context of domain expert's expectations [6]. Additionally, an important issue is that application of different clustering algorithms as well as different ways of data representation and preparation may result in substantially different clustering results.

Unfortunately, it was not possible to include experts participating in the ADNI into the analysis loop of our clustering of the ADNI data. Expert evaluation of the results solely with the help of publicly available data and their descriptions is difficult because some data or their aspects, especially anamnestic data and data collection procedures, are not public due to patient privacy protection issues. Nevertheless, we still used human-in-the-loop approach that concentrated on the identification of subpopulations that are large and interpretable with existing domain knowledge. As a result-besides some clusters that are in agreement with the existing domain knowledge-we have surprisingly identified some additional clusters that are hard to evaluate. These clusters are currently considered as potentially interesting hypotheses; their future verification on independent data might lead to new scientific insights and potentially useful medical knowledge.

The rest of the paper starts with a summary of the related work in Sect. 2 and the presentation of the data used in the analysis in Sect. 3. Section 4 describes the novel clustering methodology, including a small illustrative example. Section 5 presents the constructed clusters of ADNI patients. The clusters are described in terms of statistical properties of patients included into each cluster, together with the complete list of identification numbers of corresponding patients. In this way, the interested reader may access additional information about specific patients from the ADNI database. Medical relevance of the results, especially a possible interpretation of the unexpected clusters is discussed in Sect. 6. The quality of best biomarkers for the constructed clusters is analyzed in Sect. 7. Section 8 concludes the paper.

\section{Related work}

The approaches suitable for the identification of relations between clinical and biological properties of AD patients can be grouped into three groups. In the first one, we have statistical approaches that typically test the significance of differences between properties of AD patients and patients from the control group. Published results obtained with these methods [7, 8] clearly demonstrate the existence of relations between the values obtained by PET imaging and the clinical diagnosis. The problem with this approach is that the identified relations are non-specific and that the severity of the disease is not strongly correlated with the measured values. The major problem seems to be that the differences in biological descriptors may be a consequence of various physiological processes and that changes of both clinical and biological variables may be a normal process in the elderly population.

Other approaches are based on data mining methods. The second group comprises supervised machine learning techniques used for identification of potentially complex relations between biological properties that strongly correlate with the AD diagnosis [9]. This is typically a very powerful approach but in the AD domain it is confronted with the problem that there exist various clinical scales of dementia but none of them can be regarded as completely reliable for determining the $\mathrm{AD}$ diagnosis.

The third group comprises unsupervised clustering approaches, which are very attractive because they do not require explicit definition of the target class and the availability of a control group of patients. The results often enable novel insights into the analyzed data. A good example is the identification of pathological subtypes of the Alzheimer's disease presented in one large group characterized by the distribution of senile plaque restricted to a small number of brain regions, and a smaller group with about $15 \%$ of patients in which the lesions were more widely distributed [10]. A general problem of clustering is instability of the results that significantly depend on the used methodology and the parameters of the algorithm selected by the user [11]. Recently, it has been demonstrated that the quality of results can be significantly improved when more than one layer of input data are used $[3,4]$. The distinguishing property of the multi-layer clustering algorithm presented in this work is that-in contrast to redescription mining [3] - our algorithm does not construct descriptions of subpopulations and that-in contrast to multi-view learning [4]—it does not require statistical independence of input data layers. Additionally, the major advantage of the multi-layer clustering algorithm is that no explicit definition of the distance measure among instances (patients) is necessary and that no explicit definition of the number or size of the resulting clusters is expected from the user [12].

The first experiments with the application of the multilayer clustering in the $\mathrm{AD}$ domain have been performed on 916 patients from the ADNI database described by 10 biological and 23 clinical descriptors [13]. The results demonstrated the existence of an AD subpopulation with a surprising property of the increased intracerebral and whole brain volumes. The experiments have been repeated on a set of 659 ADNI patients described with 56 biological and 187 clinical properties collected during the baseline evaluation [14]. In spite of different datasets (though some 
of the patients and some of the features were overlapping), again a cluster of male patients has been identified with the same surprising property of increased intracerebral and whole brain volumes. This work is an extension of these experiments on the latter dataset but with an improved clustering algorithm. The improvement resulted in identification of an additional cluster of male patients with no dementia, construction of significantly larger clusters, and elimination of obvious outliers that have been present in previous subpopulations. The basic conclusions of this work are the same as those in [14] but the statistical significance of the results is higher.

\section{Data}

All experiments were performed on the data from the Alzheimer's Disease Neuroimaging Initiative (ADNI) database. ${ }^{1}$ The total number of patients initially included was 1736 from all ADNI stages of study (i.e., ADNI-1, ADNIGO, and ADNI-2). In order to achieve the broadest clinical dataset, some exams used only in ADNI-2 were used. Keeping only observations for which no missing values were present led to a reduction in the number of observations from 1736 to 659 . This subset includes 317 female and 342 male patients. The patients are described by 147 clinical variables, 41 laboratory variables, 40 symptoms, and 15 biological measurements. Clinical variables include Alzheimer's Disease Assessment Scale (ADAS13), Mini Mental State Examination (MMSE), Rey Auditory Verbal Learning Test (RAVLT immediate, learning, forgetting, percentage of forgetting), Functional Assessment Questionnaire (FAQ), Montreal Cognitive Assessment (MOCA), and Everyday Cognition, which are cognitive functions questionnaire filled out by patients (ECogPt) and their study partners (ECogSP) (Memory, Language, Visuospatial Abilities, Planning, Organization, Divided Attention, and the Total score), Neuropsychiatric Inventory Questionnaire, Modified Hachinski Ischemia Scale, and Geriatric Depression Scale. Examples of laboratory variables are red blood cells and total bilirubin, while examples of symptoms are palpitations and dizziness. Biological measurements include ABETA peptides, TAU and PTAU proteins, the APOE-related genetic variations (APGEN1 genotype allele 1, APGEN2 genotype allele 2), PET imaging results FDG-PET and AV45, MRI

\footnotetext{
1 The ADNI was launched in 2003 by the National Institute on Aging (NIA), the National Institute of Biomedical Imaging and Bioengineering (NIBIB), the Food and Drug Administration (FDA), private pharmaceutical companies and non-profit organizations. The Principal Investigator of this initiative is Michael W. Weiner, MD, VA Medical Center and University of California, San Francisco. More information can be found at http://www.adni-info.org and http://adni. loni.usc.edu.
}

volumetric data [Ventricles, Hippocampus, Whole Brain, Entorhinal, Fusiform gyrus, Middle temporal gyrus (MidTemp) and intracerebral volume (ICV)].

For the evaluation of the consistency of constructed clusters, we use global clinical dementia rating score which is interpreted as clinically normal $\mathrm{CN}$ (value 0 ), mild cognitive impairment MCI (value 0.5) and Alzheimer's disease AD (value 1) diagnosis for the patient. The clinical dementia rating score is different from the five level ADNI patient diagnosis (cognitive normal, significant memory concern, early mild cognitive impairment, late mild cognitive impairment, and $\mathrm{AD}$ ), but the agreement between the two scales is very high.

In clustering, the symmetry and additivity of the variables prove to be important. Therefore, in data preprocessing, some of the variables were transformed to achieve reduced skewness. The transformation function was selected according to the type of data measured by the variable, the level of skewness, and the most adequate function from a set of possible functions, which include $\log x, \operatorname{logit} x, 1 / x$, etc.

\section{Multi-layer clustering}

In a typical machine learning setting, we have a set of examples $E$ that are described by a set of attributes $A$, and from these examples we try to induce or learn a model that would generalize the examples. In some domains, the set of attributes may be partitioned in two or more disjoint subsets (layers) according to some criteria, such as the physical meaning of the attributes or the way data on specific attributes have been collected. For example, in the Alzheimer's disease domain, the first layer can be the laboratory data, while the second layer can be the clinical data. In some other domain, different layers may contain the same attributes but collected in various time periods. The goal of multi-layer clustering is to construct clusters that are as large as possible and coherent in all the layers. This section describes the proposed clustering methodology by first describing single-layer clustering, and then generalizing it to multi-layer clustering.

\subsection{Single-layer clustering}

Let us assume a basic clustering task in which we have only one layer of attributes. The proposed methodology consists of two steps. In the first step, we compute the socalled example similarity table. This is an $N \times N$ symmetric matrix, where $N$ is the number of examples. All its values are in the range $0.0-1.0$. A large value at a position $(i, j), i \neq j$, denotes a large similarity between examples $i$ and $j$. In the second step, we use the table in order to construct clusters. 


\subsubsection{Example similarity table (EST)}

We start from the original set of $N$ examples described by nominal and numerical attributes that may contain unknown values. An artificial classification problem is formulated as follows: the examples from the original set constitute the positive examples, while the negative examples are artificially constructed by shuffling the values of the original examples. The shuffling is performed at the level of attributes so that we randomly mix values among the examples. The values remain within the same attribute as in the original set of examples. As a result, we have the same values in positive and negative examples, but in negative examples we have randomized connections between the attribute values. For small problems with up to 200 examples, we typically construct four times as many negative examples as in the original (positive) example set, while for larger domains we construct the same number of positive and negative examples.

Next, we use a supervised machine learning algorithm to build a predictive model that is used to discriminate between the positive examples (the original examples) and the negative examples (the artificially constructed examples with shuffled attribute values). The goal of learning is not the predictive model itself, but the information on the similarity of examples. Machine learning approaches with which we can determine if some examples are classified in the same way are appropriate for this task. For example, in decision tree learning this means that examples end in the same leaf node, while in decision rule learning this means that examples are covered by the same rule.

To estimate the similarity of examples, we follow an ensemble learning approach, where statistics are computed over a large set of classifiers. Additionally, a necessary condition for a good similarity estimation is that the classifiers are as diverse as possible and that each of the classifiers is better than random. All these conditions are satisfied, e.g., by the Random Forest [15, 16] and the Random Rules [17] algorithms. We use the latter approach in which we typically construct about 50,000 rules for each EST computation.

The similarity of examples is determined so that for each pair of examples, we count how many rules cover both examples. The EST presents the statistics for the positive examples (original set of examples). A pair of similar examples will be covered by many rules, while no rules or a very small number of rules will cover pairs that are very different in terms of their attribute values. Final EST values are computed by normalizing the counts by the largest detected value.

Table 1 presents an example of EST for a set of 6 examples. In the upper part is the table with counts of rules covering pairs of examples. The diagonal elements
Table 1 An illustrative example of a similarity table (EST)

\begin{tabular}{rrrrrrr}
\hline & ex1 & ex2 & ex3 & ex4 & ex5 & ex6 \\
\hline ex1 & 38 & 0 & 27 & 28 & 0 & 7 \\
ex2 & 0 & 97 & 3 & 1 & 97 & 3 \\
ex3 & 27 & 3 & 47 & 16 & 3 & 1 \\
ex4 & 28 & 1 & 16 & 45 & 1 & 4 \\
ex5 & 0 & 97 & 3 & 1 & 97 & 3 \\
ex6 & 7 & 3 & 1 & 4 & 3 & 39 \\
\hline & ex1 & ex2 & ex3 & ex4 & ex5 & ex6 \\
\hline ex1 & 0.39 & 0.0 & 0.28 & 0.29 & 0.0 & 0.07 \\
ex2 & 0.0 & 1.0 & 0.03 & 0.01 & 1.0 & 0.03 \\
ex3 & 0.28 & 0.03 & 0.48 & 0.16 & 0.03 & 0.01 \\
ex4 & 0.29 & 0.01 & 0.16 & 0.46 & 0.01 & 0.04 \\
ex5 & 0.0 & 1.0 & 0.03 & 0.01 & 1.0 & 0.03 \\
ex6 & 0.07 & 0.03 & 0.01 & 0.04 & 0.03 & 0.40 \\
\hline
\end{tabular}

represent total counts of rules covering each example. By the normalization of this table, we obtain the EST that is presented in the lower part of the table. It can be noticed that we have two very similar examples (ex2 and ex5), three similar examples (exl, ex3, and ex4), and one very different example $(e x 6)$. The maximal value in the upper table is 97 and EST values in the lower table are obtained through normalization with this value.

\subsubsection{Clustering-related variability (CRV) score}

The second step in the process of clustering starts from the EST. The goal is to identify subsets of examples that can reduce the variability of EST values. For this purpose, we define the so-called clustering-related variability (CRV) score. This is the basic measure which guides the search during iterative bottom-up clustering. CRV score is not a simple similarity measure. It is defined for a single example, but it depends also on other examples that this example is clustered with. A cluster may consist of a single example.

Clustering-related variability, for example $i$ is denoted as $\mathrm{CRV}_{i}$. It is the sum of squared deviations of EST values in row $i\left(X_{i}=\left\{x_{i, j}, j \in\{1, \ldots, i-1, i+1, \ldots, N\}\right\}\right)$ but so that $\mathrm{CRV}_{i}$ is computed as a sum of two components: $\mathrm{CRV}_{i}=\mathrm{CRV}_{i, \mathrm{wc}}+\mathrm{CRV}_{i, \mathrm{oc}}$.

Within cluster value

$$
\mathrm{CRV}_{i, \mathrm{wc}}=\sum_{j \in C}\left(x_{i, j}-x_{\text {mean,wc }}\right)^{2}
$$

is computed as a sum over columns $j$ of row $i(j \neq i)$ corresponding to examples included in the same cluster $C$ with example $i$. In this expression, $x_{\text {mean,wc }}$ is the mean value of all $x_{i, j}$ in the cluster. When there is only one 
example in a cluster then $\mathrm{CRV}_{i, \mathrm{wc}}=0$ because there are no other examples in the cluster that are different from $i$. When there are two examples in a cluster then it equals zero because we compute the sum only for one value $x_{i, j}$ and that is equal to $x_{\text {mean,wc }}=x_{i, j}$.

Outside cluster value

$$
\mathrm{CRV}_{i, \mathrm{oc}}=\sum_{j \notin C}\left(x_{i, j}-x_{\text {mean }, \mathrm{oc}}\right)^{2}
$$

is defined in the same way as $\mathrm{CRV}_{i, \mathrm{wc}}$ but for $x_{i, j}$ values of row $i$ not included in cluster $C$. The $x_{\text {mean,oc }}$ is the mean value of the EST element values not included in the cluster and it is different from the $x_{\text {mean,wc }}$ used to compute $\mathrm{CRV}_{i, \mathrm{wc}}$. When example $i$ is the only example in a cluster then $\mathrm{CRV}_{i, \mathrm{oc}}$ is the sum of squared deviations for all the values in row $i$ except for $x_{i, i}$.

The final CRV value of cluster $C$ is computed as the sum of all the CRV values for the examples contained in the cluster:

$$
\mathrm{CRV}_{C}=\sum_{i \in C} \mathrm{CRV}_{i}
$$

\subsubsection{Illustrative example}

We use the data from the EST presented in Table 1 to compute the CRV value for the example (ex1) contained in various clusters $C$. We present three cases: when cluster $C$ contains only example ex 1 , when ex1 is clustered with $e \times 3$, and, finally, when it is clustered with both $e \times 3$ and $e \times 4$. By visual inspection of the EST, we can immediately notice some similarity among examples $\{$ ex 1, ex 3, ex 4$\}$. The goal is to demonstrate the CRV value computation to show that for the same row ex1, we can get different $\mathrm{CRV}_{e x 1}$ values depending on which example ex 1 is clustered with, and finally to show how $\mathrm{CRV}_{e x 1}$ values decrease when similar examples are added into cluster $C$.

If $e x 1$ is the only example in a cluster $C=\{e x 1\}$ :

$\mathrm{CRV}_{\text {ex } 1, \mathrm{wc}}=0$

$\mathrm{CRV}_{\text {ex } 1, \mathrm{oc}}=(0.0-0.13)^{2}+(0.28-0.13)^{2}+$

$(0.29-0.13)^{2}+(0.0-0.13)^{2}+(0.07-0.13)^{2}=0.08$

$\mathrm{CRV}_{\text {ex } 1}=0.08$

When we add a new element (ex3) to this cluster $C=\{$ ex 1, ex 3$\}:$

$\mathrm{CRV}_{e x 1, \mathrm{wc}}=(0.28-0.28)^{2}=0.00$

$\mathrm{CRV}_{\text {ex } 1, \mathrm{oc}}=(0.0-0.09)^{2}+(0.29-0.09)^{2}+$

$(0.0-0.09)^{2}+(0.07-0.09)^{2}=0.06$

$\mathrm{CRV}_{e x 1}=0.06$

Finally, when we have $C=\{e x 1, e \times 3, e x 4\}$ :

$\mathrm{CRV}_{e x 1, \mathrm{wc}}=(0.28-0.285)^{2}+(0.29-0.285)^{2}=0.00$

$\mathrm{CRV}_{\text {ex } 1, \mathrm{oc}}=(0.0-0.02)^{2}+(0.0-0.02)^{2}+$

$(0.07-0.02)^{2}=0.00$
$\mathrm{CRV}_{e x 1}=0.00$

\subsubsection{Single-layer algorithm}

Algorithm 1 is the bottom-up clustering algorithm that merges the most similar examples in respect of the CRV score, and produces a hierarchy of clusters. It may be noticed that in contrast to most other clustering algorithms, it has a well-defined stopping criterion. The process stops when further merging does not result in the reduction of example variability measured by the CRV score, and this way the algorithm automatically determines the optimal number of clusters. As a consequence, some examples may stay non-clustered (more precisely, they remain as clusters consisting of only one example).

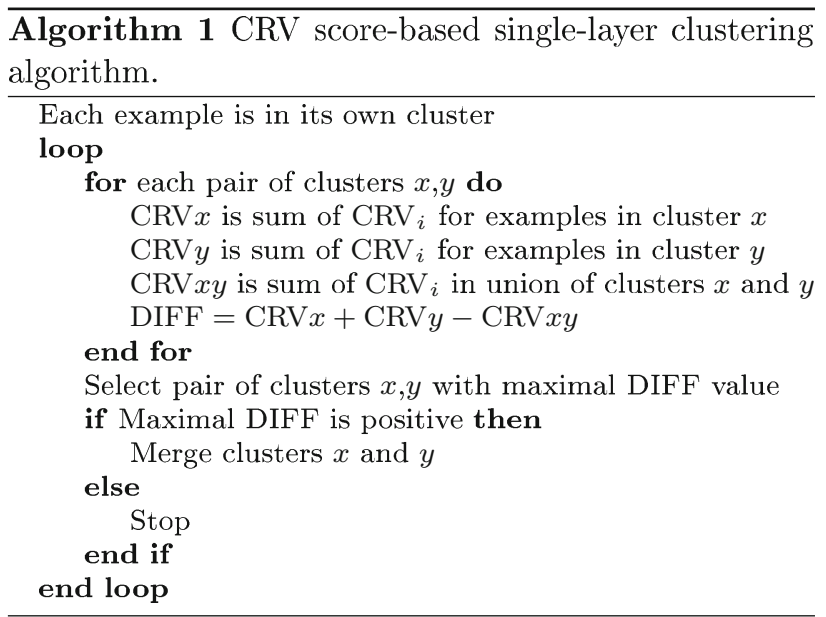

\subsection{Multi-layer clustering}

The basic lesson learned from redescription mining and multi-view clustering is that the reliability of clustering can be significantly improved by a requirement that the result should be confirmed in two or more attribute layers. The approach for clustering based on example similarity, presented in the previous section for a single-layer case, can be easily extended to clustering in a multi-layer case.

If we have more than one attribute layer then for each of them we compute the example similarity table independently. For each layer, we have to construct its own artificial classification problem and execute the supervised learning process in order to determine the similarity of examples. Regardless of the number and type of attributes in different layers, the tables will be always matrices of dimension $N \times N$. The reason is that by definition, we have the same set of $N$ examples in all the layers. After computing the similarity tables, the second step of the clustering process is executed. 


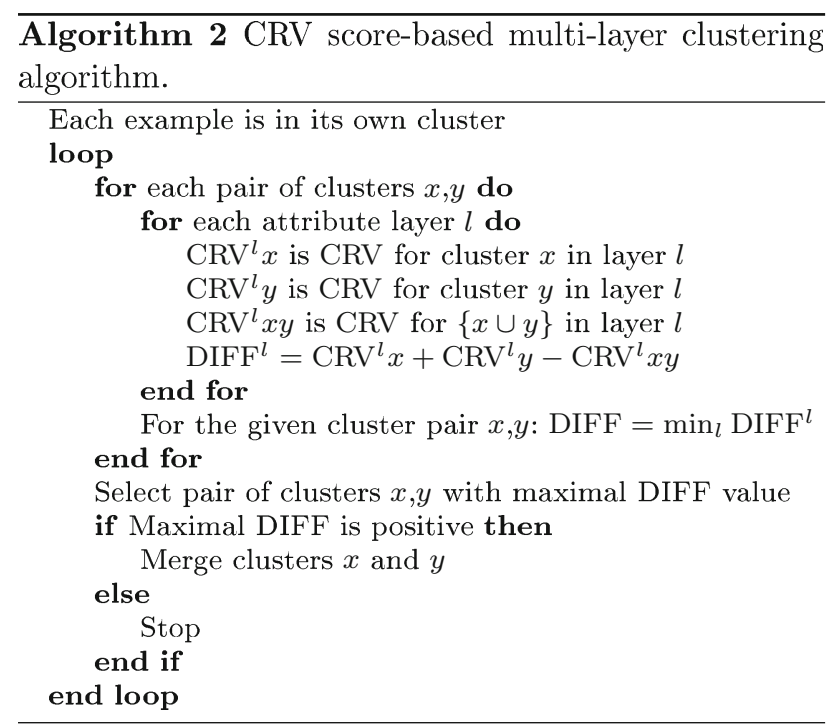

Conceptually, multi-layer clustering presented in Algorithm 2 is identical to the single-layer approach. The main difference is that merging of two clusters is possible only if there is variability reduction in all the layers. For each possible pair of clusters, we have to compute potential variability reduction for all attribute layers and to select the smallest value for this pair. If this minimal value is positive, then merging of clusters enables variability reduction in all the layers. When there are more pairs with positive minimal value, we chose the pair with the largest minimal value and merge these clusters in the current iteration.

When we do clustering in two or more layers, we have a conjunction of necessary conditions for merging two clusters. A typical consequence is that resulting clusters are smaller than in the case of a single-layer clustering.

\section{Clustering results}

Clustering was performed independently for each of the two subpopulations of 317 female and 342 male patients. A series of experiments was performed so that different parts of available information about patients were used as input layers. The presented results were obtained by using biological measurements and laboratory data (in total 56 descriptors) as the first layer, and symptoms and clinical data (in total 187 descriptors) as the second layer.

All experiments produced a large number of clusters. For the described setting with two layers for the female population, there are 19 clusters with 4 or more patients, nine of which have more than 10 patients. The result for the male population is very similar: 21 clusters with 4 or more patients, 10 of them with more than 10 patients. Five largest clusters for each population are listed in Table 2. They
Table 2 List of five largest clusters for female and male populations

\begin{tabular}{lllll}
\hline Number of patients & \multicolumn{2}{l}{ Distribution of CD rating score } & Cluster ID \\
\cline { 2 - 3 } & $\mathrm{AD}$ & $\mathrm{MCI}$ & $\mathrm{CN}$ & \\
\hline Females & & & 59 & $\mathrm{~F} 0$ \\
64 & 0 & 5 & 1 & $\mathrm{~F} 1$ \\
47 & 19 & 27 & 2 & - \\
22 & 0 & 20 & 20 & - \\
20 & 0 & 0 & 1 & - \\
19 & 3 & 15 & & \\
Males & & & 30 & M0A \\
42 & 0 & 12 & 32 & M0B \\
40 & 0 & 8 & 0 & M1 \\
38 & 18 & 20 & 0 & M2 \\
31 & 13 & 18 & 1 & - \\
27 & 0 & 26 & &
\end{tabular}

include a bit more than a half of patients from each population.

Table 3 presents the clinical and biological properties for six clusters that include more than 30 patients. For the female population, we have one large cluster F1 in which the majority of patients have significant problems with dementia. Out of the 47 included patients, 19 have the Clinical Dementia rating score equal to 1 (in this work interpreted as AD), while 28 have been diagnosed as mild cognitive impairment (CD score of 0.5). In the entire dataset, there are 22 patients with the score value equal to 1 , and 19 of them are included into this cluster. The clinical properties of these patients include high ADAS13, FAQ, and MMSE scores, and all types of cognitive problems. The biological properties of these patients are also typical for AD patients, e.g., low FDG values, significantly decreased Entorhinal volume, and high AV45 values. A statistical comparison with the population of all 145 female patients with cognitive normal status in the dataset has been used to identify the most distinguishing biological properties of the cluster. The last column of Table 3 presents the most significant properties in terms of the highest z-score values of the Mann-Whitney test. The values are very high denoting that differences between cognitive normal patients and those included in the cluster are very significant. $^{2}$

Cluster F0 constructed for the female population includes 64 patients that are typical patients with no significant problems with dementia. Although this is the largest cluster constructed for the female population, it is relatively small if we take into account that there are 145

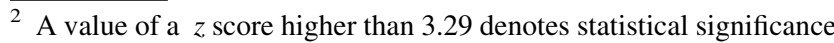
of $P<0.001$.
} 
Table 3 Short descriptions for the largest clusters

\begin{tabular}{llll}
\hline $\begin{array}{l}\text { Cluster } \\
\text { ID }\end{array}$ & Clinical status & $\begin{array}{l}\text { Biological properties } \\
\text { (with } z \text { score versus } \\
\text { cognitive normal) }\end{array}$ \\
\hline Clusters for female patients & & \\
F1 & Significant cognitive problems & Low FDG & 9.29 \\
& (high ADAS13, high FAQ, high & Low entorhinal & 8.74 \\
& MMSE) & High AV45 & 8.36 \\
& & Low & 8.33 \\
& & hippocampus \\
& & Low MidTemp & 7.52 \\
& & Low fusiform & 7.13 \\
& & High TAU & 7.12 \\
& & High FDG & 6.05 \\
F0 & Mild or no dementia & High & 2.86 \\
& & hippocampus & \\
& & High whole & 2.85 \\
& & brain &
\end{tabular}

Clusters for male patients

\begin{tabular}{|c|c|c|c|}
\hline \multirow[t]{6}{*}{ M1 } & \multirow[t]{6}{*}{ Significant cognitive problems } & Low FDG & 8.23 \\
\hline & & $\begin{array}{l}\text { Low } \\
\quad \text { hippocampus }\end{array}$ & 7.70 \\
\hline & & Low entorhinal & 7.09 \\
\hline & & Low MidTemp & 6.33 \\
\hline & & $\begin{array}{l}\text { Low whole } \\
\text { brain }\end{array}$ & 6.23 \\
\hline & & High TAU & 5.57 \\
\hline \multirow[t]{5}{*}{ M2 } & \multirow[t]{5}{*}{ Significant cognitive problems } & Low FDG & 7.83 \\
\hline & & High ICV & 6.54 \\
\hline & & High ventricles & 5.70 \\
\hline & & $\begin{array}{l}\text { Low } \\
\quad \text { hippocampus }\end{array}$ & 5.60 \\
\hline & & Low ABETA & 5.80 \\
\hline \multirow[t]{3}{*}{ MOA } & \multirow[t]{3}{*}{ Mild or no dementia } & High FDG & 4.98 \\
\hline & & High ICV & 4.00 \\
\hline & & $\begin{array}{l}\text { High whole } \\
\text { brain }\end{array}$ & 3.31 \\
\hline \multirow[t]{2}{*}{ MOB } & \multirow[t]{2}{*}{ Mild or no dementia } & Low ICV & 4.83 \\
\hline & & $\begin{array}{l}\text { Low whole } \\
\text { brain }\end{array}$ & 3.74 \\
\hline
\end{tabular}

cognitive normal female patients in the whole dataset. A possible explanation is that among ADNI patients diagnosed as cognitive normal there are also patients that are not completely healthy, but their subjective or objective problems are either not severe enough or their problems are in discrepancy with typical clinical profiles.

The bottom part of Table 3 presents clusters for the male population. There are two clusters of patients with significant cognitive problems (M1 and M2) and two clusters of patients with mild or no dementia (M0A and M0B). Cluster M0A is similar in terms of the properties of the female cluster F0 both regarding its size and the detected biological properties. It is interesting to notice that cluster MOA includes even 12 patients that have $\mathrm{CD}$ rating score equal to 0.5 while in the female cluster there are only five such patients. In contrast to cluster M0A, cluster M0B is characterized by decreased ICV values.

There are two clusters of male patients that have significant problems with dementia. In the first one (M1) there are 38 patients, 18 of them with the $\mathrm{CD}$ score equal to 1 and the rest with the score equal to 0.5 . In the second cluster (M2) there are 31 patients, 13 of them with $C D$ score equal to 1 . In the male population, there are a total of 36 patients with $\mathrm{AD}$ status ( $\mathrm{CD}$ value equal to 1 ). An interesting observation is that two male clusters M1 and M2 together include 31 out of $36(86 \%)$ male patients with $\mathrm{CD}$ score equal to 1 in the dataset, while the single female cluster F1 includes almost identical percentage of such patients (19 out of 22 , i.e., $86 \%$ ). Table 4 lists the ADNI IDs of patients included into clusters F0, F1, M0A, M0B, M1, and M2.

\section{Analysis of results}

A significant difference between male and female populations of patients can be noticed. For the female population there are two clusters while for the male population there are four clusters, two for patients with significant problems with dementia and two for patients with mild or no dementia. By inspecting the properties characterizing patients in these clusters (see Table 3), one can notice especially interesting differences between patients in clusters M1 and M2. Biological and clinical properties that most significantly differentiate these two clusters according to the Mann-Whitney test are listed in Table 5.

Cluster M2 deserves special attention due to the fact that average values of ICV and whole brain volume for patients in M2 are higher than average values for the set of all 124 cognitive normal male patients. The result is unexpected because cognitive problems are typically related with the atrophy of human brain [18]. The differences are statistically significant; average ICV values are 1,577 and 1,774 for cognitive normal and M2 patients, respectively ( $z$ score 6.54, $P<0.001$ ) (see Table 3), while average whole brain volumes are 1109 and 1167 ( $z$ score 3.08, $P<0.01){ }^{3}$ When comparing patients in cluster M2 with patients in M1, who also have typical AD symptoms but, as expected, decreased ICV and whole brain volumes, the differences are even more statistically significant (see Table 5).

\footnotetext{
${ }^{3}$ Actual absolute values for ICV and whole brain volumes are 1000 times larger.
} 
Table 4 Lists of ADNI patients included into clusters from Table 3
For clusters F1, M1, and M2, RIDs of patients with the diagnosis of the Alzheimer's disease (CDGLOBAL value equal to 1 ) are typeset in bold, while for clusters F0, M0A, and MOB, patients with the MCI diagnosis (CDGLOBAL value equal to 0.5 ) are typeset in bold

\begin{tabular}{|c|c|c|c|c|c|c|c|c|c|c|c|c|c|}
\hline \multicolumn{14}{|c|}{ Cluster F1 } \\
\hline 4024 & 4030 & 4034 & 4058 & 4079 & 4201 & 4209 & 4211 & 4252 & 4324 & 4353 & 4402 & 4415 & 4458 \\
\hline 4477 & 4500 & 4502 & 4542 & 4568 & 4591 & 4609 & 4660 & 4715 & 4796 & 4815 & 4845 & 4894 & 4897 \\
\hline 4902 & 4904 & 4905 & 4906 & 4909 & 4910 & 4912 & 4918 & 4982 & 4984 & 4990 & 4997 & 5006 & 5015 \\
\hline 5019 & 5031 & 5063 & 5119 & 5184 & & & & & & & & & \\
\hline \multicolumn{14}{|c|}{ Cluster M1 } \\
\hline 4009 & 4095 & 4096 & 4131 & 4152 & 4171 & 4195 & 4215 & 4240 & 4307 & 4475 & 4494 & 4501 & 4526 \\
\hline 4625 & 4672 & 4686 & 4689 & 4707 & 4718 & 4770 & 4774 & 4802 & 4827 & 4857 & 4867 & 4936 & 4958 \\
\hline 4964 & 4968 & 4980 & 4994 & 5017 & 5027 & 5067 & 5165 & 5224 & 5241 & & & & \\
\hline \multicolumn{14}{|c|}{ Cluster M2 } \\
\hline 4136 & 4153 & 4192 & 4223 & 4243 & 4258 & 4346 & 4423 & 4515 & 4546 & 4549 & 4595 & 4615 & 4661 \\
\hline 4692 & 4733 & 4859 & 4863 & 4924 & 4943 & 4971 & 4974 & 5012 & 5037 & 5058 & 5059 & 5070 & 5071 \\
\hline 5095 & 5208 & 5210 & & & & & & & & & & & \\
\hline \multicolumn{14}{|c|}{ Cluster F0 } \\
\hline 4028 & 4066 & 4076 & 4084 & 4155 & 4184 & 4200 & 4288 & 4320 & 4335 & 4340 & 4349 & 4357 & 4362 \\
\hline 4399 & 4401 & 4422 & 4441 & 4446 & 4483 & 4496 & 4508 & 4545 & 4553 & 4555 & 4598 & 4607 & 4624 \\
\hline 4643 & 4644 & 4645 & 4843 & 4872 & 4874 & 4878 & 4900 & 4952 & 5093 & 5102 & 5118 & 5127 & 5129 \\
\hline 5132 & 5154 & 5158 & 5159 & 5169 & 5175 & 5185 & 5193 & 5198 & 5203 & 5214 & 5230 & 5235 & 5240 \\
\hline 5261 & 5272 & 5277 & 5287 & 5288 & 5289 & 5290 & 5292 & & & & & & \\
\hline \multicolumn{14}{|c|}{ Cluster M0A } \\
\hline 4029 & 4037 & 4043 & 4082 & 4164 & 4177 & 4179 & 4210 & 4225 & 4229 & 4257 & 4274 & 4309 & 4332 \\
\hline 4339 & 4345 & 4352 & 4389 & 4427 & 4429 & 4431 & 4453 & 4485 & 4516 & 4520 & 4556 & 4604 & 4632 \\
\hline 4649 & 4739 & 4844 & 4921 & 4926 & 4941 & 4966 & 5113 & 5131 & 5141 & 5157 & 5242 & 5271 & 5296 \\
\hline \multicolumn{14}{|c|}{ Cluster M0B } \\
\hline 4086 & 4090 & 4103 & 4158 & 4168 & 4176 & 4251 & 4292 & 4369 & 4391 & 4400 & 4443 & 4464 & 4469 \\
\hline 4491 & 4577 & 4579 & 4601 & 4620 & 4762 & 4799 & 4813 & 4862 & 4877 & 5082 & 5083 & 5109 & 5130 \\
\hline 5135 & 5147 & 5150 & 5167 & 5212 & 5243 & 5248 & 5250 & 5266 & 5278 & 5279 & 5294 & & \\
\hline
\end{tabular}

The importance of the discovery is manifold. First, it indicates gender-specific differences because such a cluster with similar properties is not detected in the female population. Second, for a domain in which biological processes with opposite manifestations (decrease and increase of ICV) may result in similar clinical consequences (dementia), segmentation of the patient population is suggested before other analyses aimed at the discovery of relations between biological and clinical properties of patients are performed. Finally, the result is intriguing in respect of its biological and medical interpretation.

It is possible that the increased ICV and whole brain volumes are a consequence of an artifact in data collection procedures, feature extraction from images, or data postprocessing (normalization). The assumption may stimulate careful evaluation of the ADNI data, especially for patients in cluster M2. But the result may also suggest the existence of a different biological pathway for the male population, resulting in serious dementia problems that are often diagnosed as Alzheimer's disease but with less expressed clinical symptoms (see bottom part of Table 5). In the scientific literature, we have found no support for such explanation except that the study devoted to gender-related differences [2] concluded that "AD pathology is more likely to be clinically expressed as dementia in women than in men."

Figure 1 illustrates the differences among patients in clusters M1 and M2 and cognitive normal male patients in respect of ICV values and ADAS13 scores. It can be noticed that male cluster M0A can be compared with female cluster F0 because they share common properties: increased ICV and decreased ADAS13 score when compared to mean values of all cognitive normal male and female patients, respectively. Cluster M0B is again a surprise because it represents a group of patients which also has improved (lower) ADAS13 values but with decreased values of ICV. The differences between cluster M0B and the complete cognitive normal male patients are not statistically significant but the result additionally stresses differences between male and female populations and suggests that the differences between clusters $\mathrm{M} 1$ and $\mathrm{M} 2$ that are valid for $\mathrm{AD}$ patients are to some extent present also in the cognitive normal population. 
Table 5 Biological and clinical properties that are most significantly different for patients in clusters M1 and M2

\begin{tabular}{|c|c|c|c|c|}
\hline Property & $\begin{array}{l}\text { Average value for } \\
\text { cognitive normal males }\end{array}$ & $\begin{array}{l}\text { Average value } \\
\text { for M2 }\end{array}$ & $\begin{array}{l}\text { Average value } \\
\text { for M1 }\end{array}$ & $\begin{array}{l}\text { Mann-Whitney } z \text { score } \\
\text { M1 versus M2 }\end{array}$ \\
\hline \multicolumn{5}{|l|}{ Biological properties } \\
\hline ICV $(* 1000)$ & 1577 & 1774 & 1479 & 6.98 \\
\hline Whole brain $(* 1000)$ & 1109 & 1167 & 983 & 6.12 \\
\hline MidTemp & 21629 & 20127 & 17930 & 3.84 \\
\hline Hippocampus & 7808 & 6530 & 5722 & 3.47 \\
\hline Fusiform & 19593 & 18457 & 16672 & 3.37 \\
\hline Ventricles & 35686 & 64375 & 47414 & 2.47 \\
\hline \multicolumn{5}{|l|}{ Clinical properties } \\
\hline Abstraction_moca & 1.81 & 1.64 & 1.14 & 2.85 \\
\hline $\begin{array}{l}\text { Neuropsychiatric Inv. } \\
\text { (impatience) }\end{array}$ & 0.29 & 0.52 & 1.78 & 2.07 \\
\hline Naming_moca & 2.92 & 2.82 & 2.38 & 1.78 \\
\hline FAQTV & 0.10 & 1.97 & 2.62 & 1.62 \\
\hline
\end{tabular}

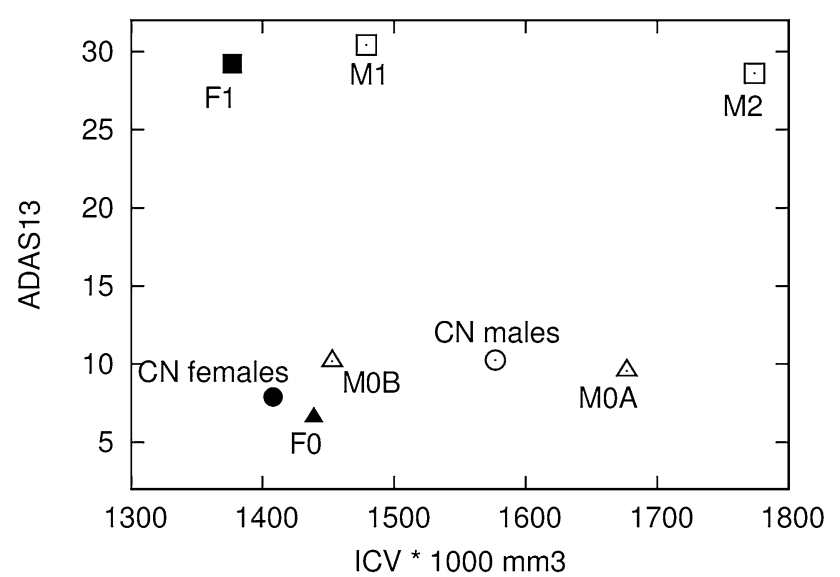

Fig. 1 Average values of Alzheimer's Disease Assessment Scale (ADAS13) and intracerebral volume (ICV) for all females with clinical dementia rating score equal zero (black circle), females in cluster F0 (black triangle), females in cluster F1 (black square), all males with clinical dementia rating score equal zero (white circle), males in clusters MOA and MOB (white triangles), and males in clusters M1 and M2 (white squares)

\section{Biological markers}

One of the stated ADNI goals is to improve clinical trial design through detection of biomarkers that could be used as approximate measures of the severity of dementia. This is known as a difficult task that is still far from a satisfactory solution. If the constructed clusters are really more homogeneous than the complete population, then it may be expected that identification of dementia disease markers should be an easier task for each cluster separately than it is for the complete population.

Table 6 presents the most correlated pairs of one biological and one clinical property that can be identified for the complete population, for the female population only, for the male population only, and finally for clusters F1, M1, and M2. The most correlated pairs are identified with the Spearman rank-order correlation coefficient $r_{s}$ that is computed for all possible pairs of properties. The result confirms that for some constructed clusters there exist more strongly correlated biological-clinical relations. ${ }^{4}$ It must be noted that in spite of a high correlation coefficient value, the statistical significance of correlation for the smallest cluster is smaller than for the larger clusters because of its size. The result means that detected high correlation is not so reliable and that it has to be confirmed by further experiments. As expected, FDG is the most useful biological property for the general patient population and the result is in agreement with previously reported research [7].

\section{Conclusions}

The presented results confirm that novel machine learning approaches to clustering can indeed be a useful tool for identifying homogeneous patient subsets in various medical knowledge discovery tasks. The applied multi-layer clustering technique and its combination with the genderrelated separation of the population of patients is definitely not the only possible approach but its results are promising. Still, significant further research effort in this direction is necessary. Clusters constructed with the multi-layer clustering are small and six largest clusters together contain only about $40 \%$ of all patients. In spite of this, the analysis of the results supports the conclusion that there are

\footnotetext{
${ }^{4}$ Only the absolute value is important, the negative sign means an inverse correlation.
} 
Table 6 Most correlated biological-clinical pairs of properties for various patient populations

\begin{tabular}{llll}
\hline Population & $\begin{array}{l}\text { Number of patients } \\
\text { Spearman correlation }\end{array}$ & Biological property & Clinical property \\
\hline All & 659 & FDG & MOCA \\
& corr. -0.51 & $(\mathrm{df}=645, P<0.001)$ & \\
Female & 317 & FDG & ADAS13 \\
& corr. -0.56 & $(\mathrm{df}=307, P<0.001)$ & \\
Male & 342 & Hippocampus & ADAS13 \\
& corr. -0.58 & $(\mathrm{df}=289, P<0.001) 1$ & \\
F1 & 47 & $\begin{array}{l}\text { MidTemp } \\
(\mathrm{df}=45, P<0.001)\end{array}$ & ADAS13 \\
& corr. -0.53 & Basophils & FAQ \\
M1 & 38 & $(\mathrm{df}=32, P<0.001)$ & \\
& corr. -0.71 & APGEN2 2 & RAVLT.forg. \\
M2 & 31 & $(\mathrm{df}=23, P<0.01)$ & \\
& corr. 0.70 & & \\
\hline
\end{tabular}

significant gender-specific differences in Alzheimer's disease. Additionally, for the male population, two subpopulations with surprising properties have been detected: A subpopulation of AD patients with increased ICV and whole brain volumes and a subpopulation of cognitive normal patients with decreased ICV volume. The result suggests that segmentation of the AD patient population is strongly recommended as a preprocessing step for any analysis aimed at understanding of relations between biological and clinical properties of $\mathrm{AD}$ patients; however, based on the available data, we still do not know how to practically perform the segmentation in a non ad-hoc manner for the majority of patients with cognitive problems.

In future work, we plan to compare multi-layer clustering with redescription mining and to test if results of redescription mining might be used for human understandable interpretation of clusters obtained by the multilayer approach. Regarding medical evaluation, we plan to test if it is possible to identify M1 and M2 clusters on nonADNI patients. The ultimate goal would be to better understand differences between these two populations.

Acknowledgments We acknowledge the European Commission's support through the Human Brain Project (Grant No. 604102), MAESTRA project (Grant No. 612944), and InnoMol project (Grant No. 316289), support of the Croatian Science Foundation (Project No. 9623: Machine Learning Algorithms for Insightful Analysis of Complex Data Structures) and support of the Slovenian Research Agency (program Knowledge Technologies and project Development and Applications of New Semantic Data Mining Methods in Life Sciences). Data collection and sharing for this project was funded by the Alzheimer's Disease Neuroimaging Initiative (ADNI) (National Institutes of Health Grant U01 AG024904) and DOD ADNI (Department of Defense award number W81XWH-12-2-0012). ADNI is funded by the National Institute on Aging, the National Institute of Biomedical Imaging and Bioengineering, and through generous contributions from many private pharmaceutical companies and nonprofit organizations.
Open Access This article is distributed under the terms of the Creative Commons Attribution 4.0 International License (http://crea tivecommons.org/licenses/by/4.0/), which permits unrestricted use, distribution, and reproduction in any medium, provided you give appropriate credit to the original author(s) and the source, provide a link to the Creative Commons license, and indicate if changes were made.

\section{References}

1. Bredesen DE (2015) Metabolic profiling distinguishing three subtypes of Alzheimer's disease. Aging 8:595-600

2. Barnes LL et al (2005) Sex differences in the clinical manifestations of Alzheimer disease pathology. Arch Gen Psychiatry 62(6):685-691

3. Galbrun E, Miettinen P (2012) From black and white to full color: extending redescription mining outside the Boolean world. Stat Anal Data Mining 5:284-303

4. Sun S (2013) A survey of multi-view machine learning. Neural Comput Appl 23:2031-2038

5. Holzinger A, Dehmer M, Jurisica I (2014) Knowledge discovery and interactive data mining in bioinformatics-state-of-the-art, future challenges and research directions. BMC Bioinform 15(suppl 6):I1

6. von Luxburg U, Williamson RC, Guyon I (2012) Clustering: science or art?. In: Proceedings of the ICML unsupervised and transfer learning, pp 65-79

7. Langbaum JB et al (2009) Categorical and correlational analyses of baseline fluorodeoxyglucose positron emission tomography images from the Alzheimer's Disease Neuroimaging Initiative (ADNI). Neuroimage 45(4):1107-1116

8. Doraiswarny PM et al (2014) Florbetapir F 18 amyloid PET and 36-month cognitive decline: a prospective multicenter study. Mol Psychiatry 19(9):1044-1051

9. Hinrichs $\mathrm{C}$ et al (2011) Predictive markers for AD in a multimodality framework: an analysis of MCI progression in the ADNI population. Neuroimage 55(2):574-589

10. Armstrong R. A. and Wood L. The identification of pathological subtypes of Alzheimer's disease using cluster analysis. Acta Neuropathol 62(6)426-432 (1994)

11. Gan G, Ma C, Wu J (2007) Data clustering: theory, algorithms, and applications. ociety for Industrial and Applied Mathematics, Philadelphia 
12. Gamberger D, Mihelčić M, Lavrač N (2014) Multilayer clustering: a discovery experiment on country level trading data. In: Proceedings of the 17th international discovery science conference, DS 2014, pp. 87-98

13. Gamberger D, Ženko B, Mitelpunkt A, Lavrač N (2015) Multilayer clustering: biomarker driven segmentation of Alzheimer's disease patient population. In: Proceedings of the international conference IWBBIO 2015, pp 134-145

14. Gamberger D, Ženko B, Mitelpunkt A, Lavrač N (2015) Identification of gender specific biomarkers for Alzheimer's disease. In: Proceedings of brain informatics and health, BIH 2015, pp 57-66

15. Breiman L (2001) Random forests. Mach Learn 45(1):5-32

16. Shi T, Horvath S (2006) Unsupervised learning with random forest predictors. J Comput Graph Stat 15(1):118-138

17. Pfahringer B, Holmes G, Wang C (2004) Millions of random rules. In: Proceedings of the workshop on advances in inductive rule learning, 15th European conference on machine learning (ECML)

18. Hample HL et al (2010) Biomarkers for Alzheimer's disease: academic, industry, and regulatory perspectives. Nat Rev Drug Discov 9:560-574

Dragan Gamberger is the Head of the Laboratory for Information Systems at the Rudjer Boskovic Institute, Zagreb, Croatia. His research interests are in machine learning with applications in intelligent data analysis. He developed algorithms for predictive rule construction, subgroup discovery, and noise detection. He received his Ph.D. in Computer Science from University of Zagreb in 1986. Together with J. Fürnkranz and N. Lavrač, he is coauthor of the book "Foundations of Rule Learning," Springer, 2012. Currently, he coordinates Croatian national project "Machine learning algorithms for insightful analysis of complex data structures." Within the project he is mainly interested in unsupervised feature selection and data clustering approaches that are based on detection of data correlations by application of supervised predictive induction.

Bernard Ženko is an Assistant Professor at the Department of Knowledge Technologies at the Jožef Stefan Institute, Ljubljana, Slovenia. He completed his Ph.D. in machine learning in 2007 at the University of Ljubljana, Slovenia, and a post-doc at the Katholieke Universiteit Leuven, Belgium in 2008. His research interests are related to machine learning and its application to practical problems from environmental and life sciences. His contributions include methods for combining multiple classifiers and learning rule-based models for predicting multi-target variables, as well as applications of machine learning methods in environmental epidemiology and medicine.

Alexis Mitelpunkt is a Senior Pediatrician and a Pediatric Neurology Resident at the Tel Aviv Medical Center and a Data Mining Researcher at the School of Statistics at the Tel Aviv University. He first has graduated with honors the Computer science studies and following that graduated at the medical school of the Sackler Faculty of Medicine at the Tel Aviv University. His residency was accomplished in pediatrics at the Dana-Dwek children's hospital at the Tel Aviv Medical Center. He has also broad experience in data mining and big data projects in various fields including health and medical applications. Currently, he is involved with Prof. Yoav Benjamini in the Medical Informatics subproject of the Human Brain EU Flagship Project.

Netta Shachar is a Statistician and a Data Scientist at the School of Statistics at the Tel Aviv University. She holds a B.Sc. (with honors) in Economics, Statistics, and Operations Research, and a M.Sc. in Applied Statistics, both from the Tel Aviv University. Her M.Sc. project was conducted under the guidance of Prof. Yoav Benjamini, within the Medical Informatics subproject of the Human Brain EU Flagship Project, where she applied statistical and machine learning tools, such as feature selection, clustering, and classification trees for the purpose of analysis of medical data.

Nada Lavrač is the Head of Department of Knowledge Technologies, Jožef Stefan Institute, Ljubljana, Slovenia. She is also a Full Professor at the University of Nova Gorica, Slovenia. She obtained a $\mathrm{Ph} . D$. in Technical Sciences from the University of Maribor, Slovenia, and was visiting scholar at Katholieke Universiteit Leuven, Leiden University, and Aalto University. Her main research interest includes machine learning and intelligent data analysis. Her research focuses on relational data mining, semantic data mining, and mining heterogeneous information networks, with applications in medicine and bioinformatics. She led the development of data mining platforms Orange4WS, ClowdFlows, and TextFlows. She is the coauthor of monographs "KARDIO: A Study in Deep and Qualitative Knowledge for Expert Systems," "Inductive Logic Programming: Techniques and Applications," and "Foundations of Rule Learning" and the editor of numerous books and conference proceedings. 